\title{
The problem of functional boundaries in prebiotic and inter-biological systems
}

\section{Leonardo Bich}

IAS-Research Centre for Life, Mind and Society. Department of Logic and Philosophy of Science. University of the Basque Country (UPV/EHU). Avenida de Tolosa 70, Donostia-San Sebastian, 20018, Spain. Email: leonardo.bich@ehu.es

\begin{abstract}
The concept of organisational closure, interpreted as a set of internally produced and mutually dependent constraints, allows understanding organisms as functionally integrated systems capable of self-production and self-maintenance through the control exerted upon biosynthetic processes and the exchanges of matter and energy with the environment. One of the current challenges faced by this theoretical framework is to account for limit cases in which a robust functional closure cannot be realised from within. In order to achieve functional sufficiency and persist, prebiotic or biological systems may need to recruit external constraints or expand their network of control interactions to include other autonomous systems. These phenomena seem to contrast with the very idea of closure and the capability of living systems to specify their functional boundaries from within. This paper will analyse from an organisational perspective the role of environmental scaffolds and of different classes of intersystem interactions in prebiotic and supra-organismal biological scenarios, and show how the theoretical framework based on the notion of closure can account for these cases.
\end{abstract}

\section{Introduction: challenges to biological autonomy}

From a system theoretical perspective, investigating the distinctive features of organisms means approaching living systems as highly integrated entities capable to control their underlying dynamics and the functional behaviour of their components in such a way as to ensure their existence and persistence over time. This line of investigation has been specifically pursued for over five decades by the theoretical framework centred on the notion of biological autonomy ${ }^{1}$. According to the autonomy framework, biological systems are organised in such a way that they realise metabolic self-production and self-maintenance. The specificity of this kind of systems is that the existence and activity of their components depend on

\footnotetext{
${ }^{1}$ For a detailed theoretical and historical analysis of this research line see Bich \& Damiano (2008), Letelier et al. (2011), and Mossio \& Bich (2017).
} 
the network they realise, and to exist as organised unities they actively manage the continuous exchange of matter and energy with the environment.

This theoretical perspective has been facing two main challenges. The first consists in characterising the distinctive dynamic regime and organisation that put together this class of systems (organisms). The second consists in specifying, on the basis of a specific theory of biological organisation, what can be considered a functional component of the system and what cannot ${ }^{2}$. A good theoretical account should be able to trace the precise functional boundaries of a system, but it might be particularly difficult to make this distinction if we consider the multifarious interactions with the environment that a living system needs to undergo and maintain in order to exist.

A detailed account of the organisation of autonomous biological systems has been recently provided in Moreno \& Mossio (2015) and Montévil \& Mossio (2015) in terms of closure of constraints. Yet, recent research on host-microbiota and, more generally, symbiotic relationships characterised by close functional ties (Bosch \& McFall-Nagai, 2011; Pradeu, 2011; Gilbert et al., 2012), might seem either to question the capability of this framework to identify clear functional boundaries for living organisms, or to call for further work of characterisation of the different ways functional interactions can be established within a system or between systems.

\section{Closure of constraints, control and functional integration.}

The notion of biological autonomy is grounded in the idea that living systems are metabolic self-producing systems that are able to self-maintain and keep their network organisation invariant through the continuous exchange of matter and energy with the environment. This idea is captured by the notion of organisational closure introduced by Jean Piaget (1967) Robert Rosen (Rosen, 1972), Humberto Maturana and Francisco Varela (Varela et al., 1974), to account for a fundamental feature of the organisation of (biological) self-maintaining systems: its circular topology as a network of processes of production of components that in turn realise and maintain the network itself. These early formulations of the notion of closure played a pioneering role in providing an understanding of the distinctive features of biological systems. Yet, they exhibited several limitations such as lack of detail, abstractness and lack of connection with thermodynamics.

Recently, an approach to closure based on the concept of constraint has been proposed to overcome these issues. Constraints are characterised as material struc-

\footnotetext{
${ }^{2}$ In this context, 'functional component' means that its existence depends on the system which harbours it, and that in turn it actively contributes to the existence of such system (Mossio et al., 2009).
} 
tures that harness processes, and that by doing so specify part of the conditions of existence of the latter. According to this framework, living systems are capable to generate a subset of the constraints acting on their internal processes, and to realise a regime of closure of constraints (Moreno \& Mossio, 2015; Montévil \& Mossio, 2015) .

A conceptual aspect of this framework that is central to discuss the functional boundaries of a living system, is that constraints play a specific functional role in a biological organisation, captured by the notion of control. ${ }^{4}$ Control is generally defined as the capability to modify the dynamics of a system toward a certain state, an activity that implies an asymmetric interaction. Specifically, in biological systems controllers are molecules or supra-molecular structures ${ }^{5}$ that are produced from within and operate as a subset of the local boundary conditions (constraints) of the controlled processes. A biological system is capable of generating some of the (internal) constraints that control its dynamics so that it can maintain itself in far from equilibrium conditions by harnessing the thermodynamic flow. An example is kinetic control (e.g., catalysis), which specifies the rates of diverse synthetic pathways: e.g. an enzyme that harnesses (catalyses) a chemical reaction, without being directly affected by it.

The distinctive feature of biological organisation, captured by the notion of $\mathrm{clo}$ sure, is that the constraints which exert these basic controls are organised in such a way that they are mutually dependent for their production and maintenance, and collectively contribute to maintain the conditions at which the whole network can persist. The notion of closure of constraints focuses, thus, on the distinctive capability of living systems to contribute to their own conditions of existence. This is a feature that is not shared by other circular networks such as abiotic water cycles (Mossio \& Bich, 2017) or self-maintaining systems such as dissipative structures, which are mostly and largely determined by external boundary conditions, and emerge spontaneously under appropriate environmental conditions.

In this perspective, control mechanisms are the functional components of a biological organisation. The way they are wired together to collectively achieve selfmaintenance provides the criteria to characterise the degree of functional integration of a system. As argued in Bich (2016), there are indeed different ways according to which control constraints can be said to be mutually dependent and realise closure. The simplest way is when control subsystems are coupled in such a way that they provide one another the substrates necessary to their own internal processes. In this case subsystems do not exert control on the others by directly af-

\footnotetext{
${ }^{3}$ For a concise discussion of the role of the notion of constraint in reframing the debate on organisational closure see Bich (2016).

${ }^{4}$ For a detailed discussion of control in biological systems see Pattee (1972) and Bich et al (2016).

${ }^{5}$ This role can be played by cellular and more complex structures in multicellular systems.
} 
fecting their local boundary conditions, but just interact by means of inputs and outputs.

The passage from functional coupling to full-fledged functional integration occurs when a control mechanism is generated by a process directly controlled by another control mechanism in the system. In this case, the two mechanisms are not simply coupled through supply and demand of metabolites, but each depends on the direct action of another constraint for its production and maintenance. They functionally contribute to one another's conditions of existence by mutually controlling their respective generative processes.

\section{Expanded functional interactions: beyond basic closure}

The ideas provided in the previous section can be employed to analyse functional interactions beyond a single autonomous system. Specifically, they can be applied to those challenging cases in which functional boundaries seem unclear and not totally specified by a regime of closure of constraints: i.e. when systems, in order to maintain themselves, need to recruit control mechanism in the environment or to establish higher order entities by exerting direct (cross) control upon other biological systems.

\subsection{Achieving functional sufficiency in prebiotic systems}

One of the conceptual issues faced by the notion of closure is whether and how it can account for cases of infra-biological (prebiotic) self-maintaining systems which might realise closure $^{6}$ without achieving full-fledged or robust autonomy. The idea is that a self -produced control network might be able to realise closure, but not to reach a stable functional sufficiency ${ }^{7}$ that allows it to persist.

Let us thinks of prebiotic scenarios of fragile self-maintaining systems: steps towards life which do not exhibit the same complexity and functional differentiation of current living systems. A hypothetical case is that of Kauffman's autocatalytic sets, a minimal theoretical example of integration realised by means of crosscontrol (Kauffman, 2000). A catalyst $A$ is generated through the action of another catalyst $B$, which controls kinetically the chemical process that leads to the syn-

\footnotetext{
${ }^{6}$ See Moreno \& Ruiz-Mirazo (2009) for a discussion of how such organisation might have been realised in the prebiotic world.

7 'Functional sufficiency' is an expression introduced by Kauffman (2016). It is used here as the set of control relationships necessary to achieve functional robustness in an organisation subject to closure.
} 
thesis of $A$. $A$, in turn, contributes to the conditions existence of $B$ by controlling directly its production or some intermediate steps, such as the synthesis of other catalysts in the system that are responsible for the production of $B$. In such a way, each constraint depends for its production and maintenance on the direct action of (at least) another control constraint in the system - a basic form of closure and they are collectively capable to realise self-production and self-maintenance.

This system is functionally integrated. Yet, it cannot generate a compartment, and therefore it does not exert (spatial) control upon some of the crucial boundary conditions that specify the medium in which its processes take place: i.e. concentrations, contiguity, permeability etc. In order for the necessary boundary conditions to be present, to avoid dispersion, to achieve the necessary crowding for reactions to take place, and therefore achieve functional sufficiency, the system can rely on environmental scaffolds for spatial control. It can do so by recruiting basic control constraints in the environment, such as micro-pores in rocks.

It would be incorrect to describe this scaffolding interaction in terms of a more comprehensive regime of functional integration, since the system does not exert any influence upon the generation of the external constraint (e.g. the pore). Yet, it does not mean that the system does not realise closure but, rather, that this kind of (hypothetical) system, although realising a basic regime of closure, is more directly determined by external boundary conditions and material constraints than other, more complex, autonomous systems.

\subsection{Integration in inter and supra-biological systems ${ }^{8}$}

Let us consider full-fledged living systems. Functional integration requires that subsystems contribute to one another's conditions of existence by mutually controlling their functional processes in such a way as to achieve closure. This concept, I will argue, allows not only to understand organisms as cohesive entities, but also to functionally account for those interactions between different organisms that are necessary for the maintenance of the organisms involved, without the need to abandon or weaken the notion of closure.

Let us first consider metabolic complementarities, such as the exchanges of metabolites and amino acids that take place between hosts and endosymbionts. In these cases a subsystem, or an organism, does not exert control on the others by affecting the local boundary conditions of their internal processes. The entities involved are mutually dependent only in a very simple form, to the extent that they provide one another the material substrates necessary for their own processes. This is not a problematic case: exchanging metabolites with the environment is inherent

\footnotetext{
${ }^{8}$ For a more detailed analysis of metabolic complementarities and cross-control between living
} systems, and their role in identifying biological individuals see Skillings and Bich (forthcoming). 
in the thermodynamic nature of biological systems. It does not require stretching or redefining the functional boundaries of the systems involved.

What does occur, instead, in those cases when functional roles are shared by different organisms? Do they call into question the notions of functional integration and closure? Let us consider three cases when the functionally integrated system is a symbiotic one or another consortium of organisms, so that control is exerted not only within but also across biological systems. For example, to respond to nutritional stress bacteria in biofilms can exchange not only metabolites, but also enzymes (or DNA sequences coding for enzymes) responsible for the control of the internal processes of other bacteria. Cross-control can be found also in arbuscolar mycorrizal symbiosis. This symbiotic relationship is realised through a mutual interaction between soil fungi and terrestrial plants, that is beneficial for both partners: the fungi receive carbon source from the plants, and the plants received other nutrients such as nitrogen and phosphate. The symbiotic interaction does not consist only in the exchange of metabolites. Actually, the exchange of nutrients is made possible by the activity of several mechanisms of control exerted by both partners on each other, within plant cells in the roots, through the development and modulation of a functional contact surface between the fungal cells and the plant cytoplasm. Another interesting phenomenon is functional replacement. The parasitic isopod cymothoa exigua enters fishes, attaches itself to the fish's tongue and causes it to fall by severing its blood vessels. Then it attaches to the stub of the tongue, and becomes the fish's new tongue. In this case a function originally exerted by a part of an organism is then exerted by a different organism

These cases are qualitatively different from ecological organisations ${ }^{9}$ - such as ecosystems, ant nests, etc. - where organisms exert control upon the external conditions of existence of other organisms, either by directly harnessing the external flux of matter and energy, or indirectly by generating external control constrains in the environment (e.g. bird nests, spider webs, beaver dams, etc.) ${ }^{10}$. In the examples described above, instead, a new order of functional integration, or an extension of an organism's functional integration, is realised because the organisms involved exert control upon one another's processes.

It is important to point out that the realisation of these new integrations does not imply per se that the organisms involved are not able to realise organisational closure and, therefore, achieve functional integration by themselves. It means, instead, that while maintaining closure as functionally cohesive entities, biological systems can extend their functional networks of control constraints by realising nested forms of functional integration that include more than one system.

\footnotetext{
${ }^{9}$ See Nunes Neto et al. (2014) for an organisational account of ecological functions.

${ }^{10}$ See also Christensen \& Bickhard (2002), for an analysis of the functional role of the bird nest in an organisational perspective.
} 


\section{Final remarks}

At first, the expansion of functional relationship to other systems - a phenomenon that occurs frequently in biology - might seem to undermine the idea of organisational closure as the basis of autonomy by blurring the distinctions between biological systems, thus undermining the possibility to understand them as functionally cohesive systems. This apparent problem depends on an incorrect interpretation of the notion of closure of constraints, which confuses the selfspecification of the functional boundaries of a living system with functional selfsufficiency. Closure is a regime of mutually dependent constraints that determines a subset of its own conditions of existence, not all of them. In this scenario, there is no problem in accounting for functional contributions that can cut across entities. A system that realises closure can undergo functional interactions with other biological systems. It can exert control upon their processes, while some of its internal processes can, in turn, be controlled by the other systems (cross-control). By establishing control interactions with other organisms, living systems do not lose their organisational closure. They also realise forms of inter-system functional integration, or possibly new super-organismal organisations, characterised by a new (higher) level of closure $^{11}$.

Acknowledgments The author thanks Derek Skillings for the stimulating discussions on metabolic complementarities and cross-control in symbiotic systems that lead to some of the ideas presented in Section 3.2. This project has received funding from the European Research Council (ERC) under the European Union's Horizon 2020 research and innovation programme - grant agreement $\mathrm{n}^{\circ} 637647$ - IDEM, from Ministerio de Ciencia, Innovación y Universidades, Spain ('Ramon y Cajal' Programme RYC-2016-19798), from Ministerio de Economia y Competitividad (MINECO), Spain (research project FFI2014-52173-P), and from the Basque Government (Project: IT 590-13).

\section{References}

Bich L. (2016). Systems and Organizations: Theoretical Tools, Conceptual Distinctions and Epistemological Implications. In G. Minati, M. R. Abram, \& E. Pessa (Eds.), Towards a Post-Bertalanffy Systemics. Springer International Publishing, New York (pp. 203-209).

Bich L., and Damiano L. (2008). Order in the nothing: Autopoiesis and the organizational characterization of the living. Electronic Journal of Theoretical Physics, 4(16), 343-373.

Bich L., Mossio M., Ruiz-Mirazo K., and Moreno A. (2016). Biological regulation: controlling the system from within. Biology \& Philosophy, 31(2), 237-265.

Bosch T. C. G., and McFall-Nagai M. J. (2011). Metaorganisms as the new frontier. Zoology, 144(4), 185-190.

Christensen W., \& Bickhard M. (2002). The process dynamics of normative function. The Monist, 85(1), 3-28.

\footnotetext{
${ }^{11}$ See Montévil and Mossio, 2015.
} 
Gilbert S. F., Sapp J., and Tauber A. I. (2012). A Symbiotic View of Life: We Have Never Been Individuals. The Quarterly Review of Biology, 87(4), 325-341.

Kauffman S. A. (2000). Investigations. Oxford University Press, New York.

Kauffman S. A. (2016). Humanity in a Creative Universe. Oxford University Press, New York.

Letelier J.-C., Cárdenas M. L., and Cornish-Bowden A. (2011). From L'Homme Machine to metabolic closure: steps towards understanding life. Journal of Theoretical Biology, 286(1), $100-13$.

Montévil M., and Mossio M. (2015). Biological organisation as closure of constraints. Journal of Theoretical Biology, 372, 179-191.

Moreno A., and Ruiz-Mirazo K. (2009) The problem of the emergence of functional diversity in prebiotic evolution. Biology \& Philosophy, 24(5):585-605.

Moreno A., and Mossio M. (2015). Biological Autonomy: a philosophical and theoretical enquiry. Springer, Dordrecht.

Mossio M., and Bich L. (2017). What makes biological organisation teleological? Synthese, 194(4), 1089-1114.

Mossio M., Saborido C., and Moreno, A. (2009). An Organizational Account of Biological Functions. The British Journal for the Philosophy of Science, 60(4), 813-841.

Nunes-Neto N., Moreno A., and El-Hani C. N. (2014). Function in ecology: An organizational approach. Biology \& Philosophy, 29(1), 123-141.

Pattee H. H. (1972). The nature of hierarchical controls in living matter. In R. Rosen (Ed.), Foundations of Mathematical Biology Volume I Subcellular Systems. Academic Press: New York (pp. 1-22).

Piaget J. (1967). Biologie et Connaissance. Gallimard, Paris.

Pradeu T. (2011). A Mixed Self: The Role of Symbiosis in Development. Biological Theory, 6, $80-88$.

Rosen R. (1972). Some relational cell models: The metabolism-repair systems. In R. Rosen (Ed.), Foundations of Mathematical Biology. Volume II Cellular Systems. Academic Press: New York (pp. 217-253).

Skillings D. and Bich L. (forthcoming). Syntrophic and Physiological Individuality.

Varela F. G., Maturana H. R., and Uribe R. (1974). Autopoiesis: The organization of living systems, its characterization and a model. Biosystems, 5(4), 187-196. 